\title{
Errata
}

Erratum: Existence of a second-order phase transition in a two-dimensional $\phi^{4}$ field theory [Phys. Rev. D 13, 2778 (1976)]

Shau-Jin Chang

There is a misprint in Eq. (A6) of the Appendix. The correct equation should read

$$
V\left(\phi_{c}, m^{\prime}\right) \equiv\langle H\rangle_{m^{\prime}} / L=\frac{1}{2} m^{2} \phi_{c}{ }^{2}+\frac{1}{4} g \phi_{c}{ }^{4}-B \phi_{c}+\frac{1}{8 \pi}\left(m^{\prime 2}-m^{2}\right)+\frac{1}{2}\left(m^{2}+3 g \phi_{c}{ }^{2}\right) \frac{1}{4 \pi} \ln \frac{m^{2}}{m^{\prime 2}}+\frac{3 g}{4}\left(\frac{1}{4 \pi} \ln \frac{m^{2}}{m^{\prime 2}}\right)^{2} \text {. }
$$

\section{Erratum: Possibility of a static scalar field in the Schwarzschild geometry}

[Phys. Rev. D 15, 1427 (1977)]

\section{R. F. Sawyer}

In Fig. 1, the abscissa should be labeled $\lambda^{1 / 2} r_{0}\left(r_{1}-r_{0}\right)^{1 / 2} r_{1}^{-1 / 2} \varphi\left(r_{1}\right)$. The ordinate should be labeled $-\left.\lambda^{1 / 2} r_{0}(d / d r)\left[\left(r-r_{0}\right)^{1 / 2} r^{-1 / 2} \varphi(r)\right]\right|_{r=r_{1}}$.

In Fig. 2, the ordinate should be labeled $\lambda^{1 / 2} r_{0}\left(r-r_{0}\right)^{1 / 2} r^{-1 / 2} \varphi(r)$. 